\title{
Differential expression of EBV proteins LMP1 and BHFR1 in EBV-associated gastric and nasopharyngeal cancer tissues
}

\author{
AILIANG WANG $^{1,2}$, WEI ZHANG ${ }^{2}$, MENG JIN $^{2}$, JIANBO ZHANG ${ }^{3}$, \\ SHENG LI $^{3}$, FENG TONG ${ }^{3}$ and YANBING ZHOU ${ }^{1}$ \\ ${ }^{1}$ Department of General Surgery, The Affiliated Hospital of Medical College of Qingdao University, \\ Qingdao, Shandong 266003; ${ }^{2}$ Department of General Surgery, The Affiliated Hospital of Jining Medical University, \\ Jining, Shandong 272000; ${ }^{3}$ Department of General Surgery, Shandong Tumor Hospital, Jinan, Shandong 250117, P.R. China
}

Received February 5, 2015; Accepted January 29, 2016

DOI: $10.3892 / \mathrm{mmr} .2016 .5087$

\begin{abstract}
Epstein-Barr virus (EBV) infection is associated with the development of $\mathrm{T}$ cell lymphoma, nasopharyngeal cancer (NPC), and EBV-associated gastric cancer (EBVaGC). This study assessed the expression of the EBV-associated proteins latent membrane protein 1 (LMP1) and BamHI-A rightward frame 1 (BARF1) in NPC and EBVaGC tissue specimens and determined their association with clinicopathological data, microvessel density (MVD) and micro-lymphatic vessel density (MLVD). This study collected 600 gastric cancer and 75 NPC tissue samples. EBV infection was assessed using in situ hybridization, and LMP1 and BARF1 expression was assessed using immunohistochemistry. The levels of MVD and MLVD were assessed using immunostaining of vascular endothelial growth factor (VEGF)-C, CD34, and lymphatic vessel endothelial receptor 1 (LYVE-1). Among the 600 gastric cancer cases, 30 were positive for EBV infection, which was shown to be associated with the age of patients $(\mathrm{P}=0.073)$, tumor differentiation $(\mathrm{P}<0.0001)$, tumor location $(\mathrm{P}<0.0001)$ and lymph node metastasis $(\mathrm{P}<0.0001)$. In these $30 \mathrm{EBVaGC}$ cases, only one case was weakly positive for LMP1, but 17 cases were BARF1 positive. BARF1 expression was associated with lymph node metastasis of EBVaGC and the level of MLVD. Furthermore, 61 (81\%) of 75 NPC patients were EBV positive, among which 38 cases were LMP-1 positive (62.3\%) and LMP1 expression was associated
\end{abstract}

Correspondence to: Dr Yanbing Zhou, Department of General Surgery, The Affiliated Hospital of Medical College of Qingdao University, 16 Jiangsu Road, Qingdao, Shandong 266003, P.R. China E-mail: zhouyanbing999@aliyun.com

Dr Feng Tong, Department of General Surgery, Shandong Tumor Hospital, 440 Jiyan Road, Jinan, Shandong 250117, P.R. China

E-mail: sdywyjy@126.com

Key words: Epstein-Barr virus infection, nasopharyngeal cancer, Epstein-Barr virus-associated gastric cancer, biomarker, microvessel density, micro-lymphatic vessel density, latent membrane protein 1, Bam $\mathrm{HI}-\mathrm{A}$ rightward frame 1 with tumor-node-metastasis stage $(\mathrm{P}=0.011)$ and lymph node metastasis $(\mathrm{P}=0.041)$. MLVD was significantly higher in LMP1-positive cases than LMP1-negative cases. There were only $8(13.3 \%)$ cases positive for BARF1 expression. In conclusion, EBV infection exhibits a role in gastric cancer and NPC development; however, expression of EBV-associated proteins LMP1 and BARF1 have differential functions during tumorigenesis of these two types of cancer.

\section{Introduction}

Epstein-Barr virus (EBV) infection contributes to human carcinogenesis, particularly in the development of lymphoma, nasopharyngeal cancer (NPC) and gastric cancer (1). EBV is a $\gamma$-herpes virus and infects $B$ cells of the immune system and epithelial cells. EBV latently persists in the host B cells for life (2) and thus, EBV is estimated to infect $>90 \%$ of the population worldwide (2). In a small number of infected cell populations, EBV infection can transform B cells to lymphoma cells (3) or epithelial cells to nasopharyngeal cancer cells (4). Furthermore, $\sim 10 \%$ of gastric cancer worldwide was associated with EBV infection and this subtype of gastric cancer is termed EBV-associated gastric cancer (EBVaGC) $(5,6)$. However, the molecular mechanism by which EBV infection causes these malignancies remains to be defined.

Molecularly, the BamHI-A rightward frame 1 (BARF1) is an EBV gene that is expressed early in the EBV lytic cycle and shares $38 \%$ primary amino acid sequence homology with the bcl-2 proto-oncogene product (7). The constitutive expression of BARF1 protein was able to immortalize lymphoblast cells and prolong cell survival (8). The carcinogenic activity of the oncogene c-myc is obligatory for the development of Burkitt lymphoma and previous studies suggested that BARF1 expression is required to inhibit c-myc-induced apoptosis and exhibits a synergic role in mediating the effect of Bcl-2 and c-myc during B cell transformation (9-13). BARF1 protein is also involved in the regulation of microvessel density (MVD) and micro-lymphatic vessel density (MLVD) $(14,15)$. Furthermore, MVD and MLVD are strongly associated with cancer metastasis and with the survival of cancer patients (14). In addition, during EBV latency, there are $>8 \mathrm{EBV}$ encoded proteins and several non-coding RNAs expressed in cells (e.g., 
two EBV encoded small RNAs, termed EBER1 and EBER2), nuclear antigens and membrane proteins (16). Latent membrane protein 1 (LMP1) and LMP2A are two EBV latent membrane proteins, which function as constitutively active receptors independent of ligand binding and manipulate the signaling pathways for B cell activation and differentiation in order to sustain the long-life of EBV-positive cells (17). The carboxyl terminus of LMP1 contains consensus tumor necrosis factor receptor-associated factor (TRAF)-binding domains, which can constitutively activate signal transducers and activators of transcription (STAT), janus kinase (JNK), and nuclear factor (NF) $\kappa$ B pathways for cell survival and growth (18). Although, the oncogenic role of LMP1 is well established, its roles in microvessel and micro-lymphatic vessel formation are less clear. Thus, the present study assessed the EBV infection in NPC and gastric cancer tissue samples, and then analyzed the levels of MVD and MLVD to identify their association with the clinicopathological features of the patients. This study may provide a novel insight into the oncogenic role of EBV in NPC and gastric cancer.

\section{Patients and methods}

Patients and tissue samples. A total of 600 gastric cancer and 75 NPC tissues were collected. All tumor tissue specimens were histologically confirmed and retrieved from the Department of Pathology, The Shandong Provincial Institute of Cancer Prevention and Research (Jinan, China) and The Affiliated Hospital of Jining Medical College (Jining, China) between 2008 and 2012. The present study was approved by the Institutional review boards of The Shandong Provincial Institute of Cancer Prevention and Research and The Affiliated Hospital of Jining Medical College. All patients provided informed consent to participate in this study.

Tissue specimens from each patient were fixed in $10 \%$ buffered formalin and embedded in paraffin (Shijiazhuang Chemical Technology Co., Ltd., Shijiazhuang, China), and then cut into serial sections $(5 \mu \mathrm{m})$. One of the consecutive sections was stained with hematoxylin and eosin (H\&E; Shanghai Biological Technology Co., Ltd., Shanghai, China) for confirmation of diagnosis, while others were subjected to in situ hybridization to detect EBV RNA or immunohistochemistry to detect the expression of LMP1, BARF1, vascular endothelial growth factor-C (VEGF-C), lymphatic vessel endothelial hyaluronan receptor-1 (LYVE-1) and CD34.

In situ hybridization and immunohistochemistry. For in situ hybridization, an ISH-5022 EBER kit was used (Beijing Zhongshan Golden Bridge Biotechnology Co., Ltd.; OriGene Technologies, Inc., Beijing, China). A labeled oligonucleotide probe complementary to EBER1 was used to detect the EBER1 as positive REBV infection. Briefly, tissue sections were deparaffinized and rehydrated, and then hybridized to a labeled probe according to the manufacturer's instructions. Tissue sections without probe hybridization were used as negative controls. For immunohistochemistry, tissue sections were deparaffinized by 2 times incubation in xylenol for $10 \mathrm{~min}$ at room temperature and dehydrated in a series of ethanol $(100,75$ and $50 \%)$. Sections were then incubated in $3 \% \mathrm{H}_{2} \mathrm{O}_{2}$ for $10 \mathrm{~min}$ at room temperature and subsequently subjected twice to antigen repair in $0.01 \mathrm{M}$ acid buffer $(\mathrm{pH}$ 6.0) using a microwave at $92-98^{\circ} \mathrm{C}$ for $5 \mathrm{~min}$, with a $10 \mathrm{~min}$ break between incubations. Sections were washed in phosphate-buffered saline (PBS), incubated with normal goat serum for $15 \mathrm{~min}$ at room temperature, and further incubated with primary antibodies at $4^{\circ} \mathrm{C}$ overnight. The primary antibodies used were rabbit monoclonal anti-LMP1 (cat. no. ZM-0386), rabbit monoclonal anti-BHRF1 (cat. no. ZA-0627), rabbit monoclonal anti-VEGF-C (cat. no. ZA-0266), mouse monoclonal anti-LYVE-1 (cat. no. ZA-0483) and mouse monoclonal anti-CD34 (cat. no. ZM-0046) (dilution used for all was 1:200; Zhongshan Golden Bridge Biotechnology Co., Ltd.; OriGene Technologies, Inc.). The sections were washed 3 times with PBS and incubated with biotinylated anti-mouse (cat. no. BA1001) or anti-rabbit (cat. no. BA1003) secondary antibodies (dilution used for all was 1:200; Boster Biological Technology, Ltd., Beijing, China) for $30 \mathrm{~min}$ at room temperature, and subsequently visualized by incubation of tissue sections with a 3,3'-diaminobenzidine solution (Zhongshan Golden Bridge Biotechnology Co., Ltd.; OriGene Technologies, Inc.). Sections were counterstained with H\&E, mounted with a coverslip and visualized using the Olympus CX23 light microscope (Olympus Corporation, Tokyo, Japan).

Review and scoring of stained tissue sections. All tissue samples were reviewed and scored blindly by two pathologists, and the pathology of each tissue section was confirmed. To assess the in situ hybridization data, negative controls were indicated as the sections without any staining, and positive controls were indicated as the stained sections with appropriate nuclear localization. To score immunohistochemical data, the two pathologists reviewed at least ten $x 400$ fields and counted staining intensity and percentage of positive cells vs. total cells. The staining intensity was judged as no staining, -; light brown, +; brown, ++; and strong brown, +++. The percentage of positive cells $<10 \%$ was considered as negative and the detailed information is as follows: $<10 \%,-; 11-25 \%$, $+; 26-50 \%,++;>50 \%,+++$. These two scores were added together to form a total score, high (score $\leq 2+$ ) vs. low expression of a protein.

Quantitative measurement of MVD and MLVD levels in tissue specimens. MVD and MLVD were visualized using immunostaining of the tissue sections with CD34 and LYE-1 antibodies, respectively, to perform morphometric analysis. The stained sections were reviewed and scored by two pathologists in a blind manner, under a CX23 light microscope. Briefly, the pathologists reviewed the sections under a x100 magnification and then selected 5 high power fields (x400 magnification) to capture images. CD34 immunostaining was used to visualize microvascular endothelial cells, while LYVE-1 is specifically expressed in microlymphatic endothelial cells. Under the CX23 light microscope, any brown colored endothelial cells or microvascular endothelial cells observed in a cluster was counted as one microvessel. Their branches were also counted as a microvessel as long as the structures were not connected. However, if the lumen had $>8$ red blood cells or the vessel had a muscular and vascular wall, this vessel was not counted. MVD was calculated as the mean number of microvessels from five x200 microscopic fields. Furthermore, micro-lymphatic 
Table I. Association of EBV infection with clinicopathological characteristics from patients with gastric cancer.

\begin{tabular}{|c|c|c|c|c|c|}
\hline Factor & $\begin{array}{l}\text { No. of cases } \\
\quad(n=600)\end{array}$ & $\begin{array}{c}\text { No. of } \\
\text { EBV-positive } \\
\text { cases }(n=30)\end{array}$ & $\begin{array}{c}\text { No. of } \\
\text { EBV-negative } \\
\text { cases }(n=570)\end{array}$ & $\chi^{2}$ & P-value \\
\hline Gender & & & & & 0.049 \\
\hline Male & 384 & 25 & 359 & 3.87 & \\
\hline Female & 216 & 5 & 211 & & \\
\hline Age (years) & & & & & 0.073 \\
\hline$<45$ & 32 & 4 & 32 & 2.24 & \\
\hline $45-60$ & 223 & 13 & 210 & & \\
\hline$>60$ & 345 & 13 & 334 & & \\
\hline Tumor differentiation & & & & & $<0.0001$ \\
\hline Well & 68 & 8 & 60 & 15.69 & \\
\hline Moderate & 124 & 12 & 112 & & \\
\hline Poor & 408 & 10 & 398 & & \\
\hline Tumor location & & & & & $<0.0001$ \\
\hline Gastric cardia region & 102 & 12 & 90 & 14.99 & \\
\hline Gastric body region & 139 & 10 & 129 & & \\
\hline Gastric antral region & 359 & 8 & 351 & & \\
\hline Lymph node metastasis & & & & & 0.0001 \\
\hline Yes & 580 & 21 & 559 & 40.39 & \\
\hline No & 20 & 9 & 11 & & \\
\hline
\end{tabular}

EBV, Epstein-Barr virus.
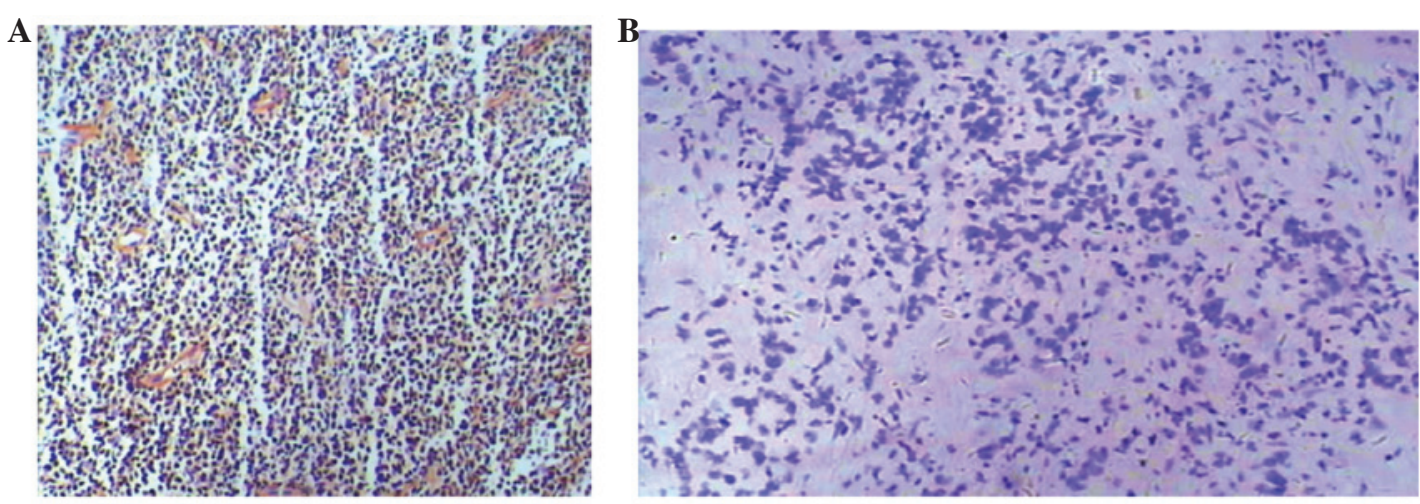

Figure 1. In situ hybridization detection of EBV-encoded small RNA 1 to indicate Epstein-Barr virus infection in gastric cancer and nasopharingeal carcinoma tissues. (A) Positive and (B) negative expression.

vessels were labeled by anti-LYVE-1 antibody and reviewed and counted as same as MVD.

Statistical analysis. All statistical analyses were performed using SPSS software, version 18.0 (SPSS Inc., Chicago, IL, USA). All data were analyzed using the Student's t-test. $\mathrm{P}<0.05$ was considered to indicate a statistically significant difference. Count data were presented as frequency and percentage, and analyzed by $\chi^{2}$ analysis or Fisher's exact test. Measurement data were presented as the mean/median \pm standard deviation. Data with normal distribution were analyzed by an F-test or t-test. Data with uncommon distribution were analyzed by a non-parametric test or Wilcoxon rank test.

\section{Results}

$E B V$ infection is associated with clinicopathological characteristics of patients. EBV infection has been linked to EBVaGC and NPC $(5,19)$; thus, in situ hybridization was performed to detect EBV-encoded small RNA 1 (EBER1) as the indication of EBV infection. Among the 600 gastric cancer tissue specimens, 30 positive cases of EBV infection were identified (Fig. 1A and Table I). Compared with negative staining, ISH positive particles had deep purple blue staining present in the nuclei (Fig. 1B). EBV infection was shown to be correlated with the age of the patient $(\mathrm{P}=0.073)$, tumor differentiation $(\mathrm{P}<0.0001)$, tumor location $(\mathrm{P}<0.0001)$ and 
Table II. Association of EBV infection with clinicopathological characteristics of patients with nasopharingeal carcinoma.

\begin{tabular}{|c|c|c|c|c|c|}
\hline Factor & $\begin{array}{l}\text { No. of cases } \\
\qquad(n=75)\end{array}$ & $\begin{array}{c}\text { No. of } \\
\text { EBV-positive } \\
\text { cases }(n=61)\end{array}$ & $\begin{array}{c}\text { No. of } \\
\text { EBV-negative } \\
\text { cases }(n=14)\end{array}$ & $\chi^{2}$ & P-value \\
\hline Gender & & & & & 0.79 \\
\hline Male & 59 & 50 & 9 & 0.070 & \\
\hline Female & 16 & 11 & 5 & & \\
\hline Age (years) & & & & & 0.311 \\
\hline$<45$ & 17 & 14 & 3 & 2.333 & \\
\hline $45-60$ & 36 & 35 & 1 & & \\
\hline$>60$ & 22 & 11 & 11 & & \\
\hline \multicolumn{6}{|l|}{ Pathology } \\
\hline Keratinizing squamous cell carcinoma & 8 & 7 & 10 & 0.016 & 0.90 \\
\hline Non-keratinizing carcinoma & 67 & 54 & 24 & & \\
\hline
\end{tabular}

EBV, Epstein-Barr virus.

Table III. Association of MVD and MLVD levels with clinicopathological characteristics of patients with Epstein-Barr-associated gastric cancer.

\begin{tabular}{lrccc}
\hline Factor & $\mathrm{n}$ & MVD & P-value & MLVD \\
\hline Gender & & & 0.408 & $3.1 \pm 0.9$ \\
$\quad$ Male & 25 & $32 \pm 2.3$ & & $3.1 \pm 1.4$ \\
Female & 5 & $33 \pm 3.1$ & 0.402 & $2.5 \pm 0.9$ \\
TNM stage & 10 & $32 \pm 3.1$ & & $3.5 \pm 1.9$ \\
I/II & 20 & $33 \pm 3.0$ & 0.111 & 3.128 \\
III/IV & & & & 3.000 \\
Lymph node metastasis & 9 & $32 \pm 3.4$ & $34 \pm 2.9$ & $3.0 \pm 1.7$ \\
Negative & 21 & & & 1.000 \\
Positive & & & & \\
\hline
\end{tabular}

MVD, microvessel density; MLVD, micro-lymphatic vessel density; TNM, tumor-node-metastasis.

lymph node metastasis $(\mathrm{P}<0.0001$; Table I). The data suggest that EBV infection only occurs in a small percentage of gastric cancers $(5 \%)$.

Furthermore, in 75 patients with NPC, 61 patients were positive for EBV infection (Table II). EBV infection was not shown to be correlated with any of the clinicopathological characteristics investigated (Table II).

Expression of the EBV-associated proteins LMPI and BHFRI is correlated with MLVD rather than MVD in patients with EBVaGC. Expression of the EBV-associated proteins LMP1 and BHFR1 and markers of MVD and MLVD (CD34 and LYVE-1) was then further analyzed in the tissue specimens. Morphometric image analysis of CD34 and LYVE-1 was used to visualize MVD and MLVD. The data showed that MVD and MLVD were not associated with TNM stage and lymph node metastasis in patients with EBVaGC (Table III). In the 30 patients with EBVaGC, only one case showed weak LMP1 expression, but 17 cases (56.7\%) showed BARF1 expression
(Fig. 2 and Table IV). BARF1 expression was significantly associated with lymph node metastasis of EBVaGC. Among the 30 patients with EBVaGC, 18 patients $(60 \%)$ were VEGF-C positive (data not shown). The expression of VEGF-C was not associated with lymph node metastasis. BARF1 expression was associated with MLVD but not MVD. These data suggest that EBV could infect lymphatic vessels and induce micro-lymphatic vessel formation. Expression of VEGF-C was associated with MVD and MLVD (data not shown).

Expression of the EBV-associated proteins LMP1 and BHFRI was correlated with MLVD rather than MVD in patients with $N P C$. Among $61 \mathrm{EBV}$ positive NPC patients, there were 38 cases that were LMP1-positive (62.3\%, Fig. 3). LMP1 expression was associated with TNM stage $(\mathrm{P}=0.021)$ and lymph node metastasis $(\mathrm{P}=0.046)$. By contrast, $\mathrm{BARF} 1$ was only expressed in 8 cases $(13.3 \%)$ and BARF1 expression was not identified to be associated with the analyzed factors. Moreover, VEGF-C was expressed in 52 cases (85.2\%) and VEGF-C expression 


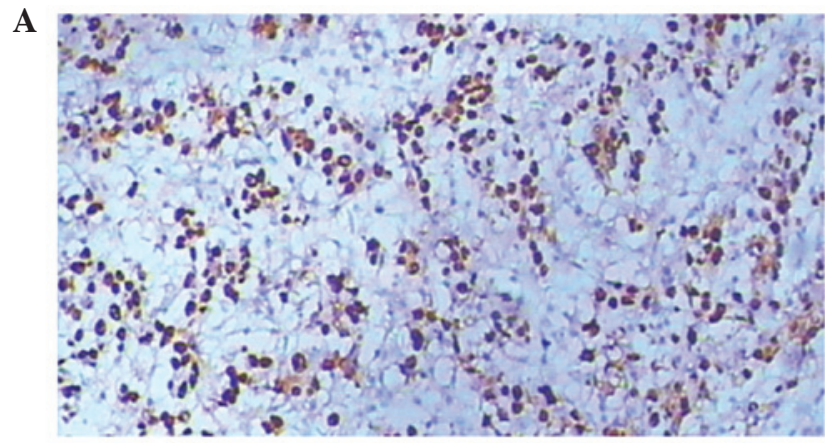

BHFR

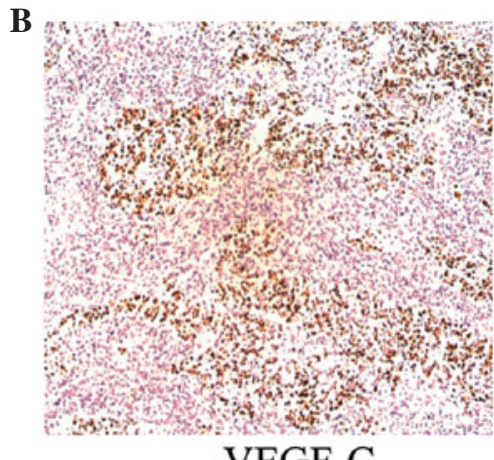

VEGF-C

Figure 2. Expression of BARF1 and VEGF-C in Epstein-Barr-associated gastric cancer (x100 magnification). (A) Positive expression of BARF1; (B) Positive expression of VEGF-C. VEGF-C, vascular endothelial growth factor-C, BARF1, BamHI-A rightward frame 1.

Table IV. Association of BARF1 and VEGF-C expression with MVD and MLVD level in Epstein-Barr-associated gastric cancer.

\begin{tabular}{lcccc}
\hline Expression & $\mathrm{n}$ & MVD & P-value & MLVD \\
\hline BARF1 & & & 1.000 & 0.000 \\
Negative & 13 & $29 \pm 3.1$ & & $1.0 \pm 0.6$ \\
Positive & 17 & $29 \pm 2.3$ & 0.000 & $3.4 \pm 0.9$ \\
VEGF-C & 12 & $23 \pm 4.8$ & & 0.000 \\
Negative & 18 & $31 \pm 4.1$ & $3.1 \pm 0.4$
\end{tabular}

BARF1, BamHI-A rightward frame 1; VEGF-C, vascular endothelial growth factor-C; MVD, microvessel density; MLVD, micro-lymphatic vessel density.

Table V. Association of BHRF1, LMP1 and VGGF-C expression with the clinicopathological data of patients.

\begin{tabular}{|c|c|c|c|c|c|c|c|c|c|c|}
\hline \multirow[b]{2}{*}{ Factor } & \multirow[b]{2}{*}{$\mathrm{n}$} & \multicolumn{3}{|c|}{ LMP1 expression } & \multicolumn{3}{|c|}{ VEGF-C expression } & \multicolumn{3}{|c|}{ BHRF1 expression } \\
\hline & & Negative & Positive & P-value & Negative & Positive & P-value & Negative & Positive & P-value \\
\hline Gender & & & & 0.931 & & & 0.893 & & & 0.914 \\
\hline Male & 50 & 20 & 30 & & 7 & 43 & & 43 & 7 & \\
\hline Female & 11 & 3 & 8 & & 2 & 9 & & 10 & 1 & \\
\hline TNM stage & & & & 0.021 & & & 0.31 & & & 0.943 \\
\hline $\mathrm{I} / \mathrm{II}$ & 27 & 18 & 9 & & 6 & 21 & & 23 & 4 & \\
\hline III/IV & 34 & 5 & 29 & & 3 & 31 & & 30 & 4 & \\
\hline LN metastasis & & & & 0.046 & & & 0 & & & 0.752 \\
\hline Negative & 14 & 10 & 2 & & 7 & 7 & & 12 & 2 & \\
\hline Positive & 47 & 13 & 36 & & 2 & 45 & & 41 & 6 & \\
\hline
\end{tabular}

LMP1, latent membrane protein 1; VEGF-C, vascular endothelial growth factor-C; BARF1, BamHI-A rightward frame 1; TNM, tumor-node-metastasis; LN, lymph node.

was associated with lymph node metastasis $(\mathrm{P}<0.0001)$. The MVD level was not shown to be significantly different between LMP1-positive and -negative cases (Tables V and VI). However, MLVD in the LMP1-positive group was significantly higher than the LMP1-negative group. This suggests that LMP1 may contribute to micro-lymphatic formation. MVD and MLVD in the VEGF-C-positive group were higher than in the negative group suggesting that VEGF-C may contribute to microvessel and micro-lymphatic formation in NPC.

\section{Discussion}

EBVaGC is a recently identified cancer type that may be caused by EBV infection (19). The present study further 
Table VI. Association of LMP1 and VEGF-C expression with MVD and MLVD levels in nasopharyngeal carcinoma.

\begin{tabular}{lcccc}
\hline Expression & $\mathrm{n}$ & MVD & P-value & MLVD \\
\hline LMP1 & & & 1.000 & 0.046 \\
Negative & 23 & $59 \pm 3.1$ & $5.0 \pm 2.0$ & $6.4 \pm 2.9$ \\
Positive & 38 & $59 \pm 5.3$ & 0.000 & 0.000 \\
VEGF-C & 9 & $53 \pm 4.8$ & & $8.1 \pm 1.8$ \\
Negative & 52 & $61 \pm 4.1$ & & $8.1 \pm 3.0$ \\
Positive & & & \\
\hline
\end{tabular}

LMP1, latent membrane protein 1; VEGF-C, vascular endothelial growth factor-C; MVD, microvessel density; MLVD, micro-lymphatic vessel density.

A

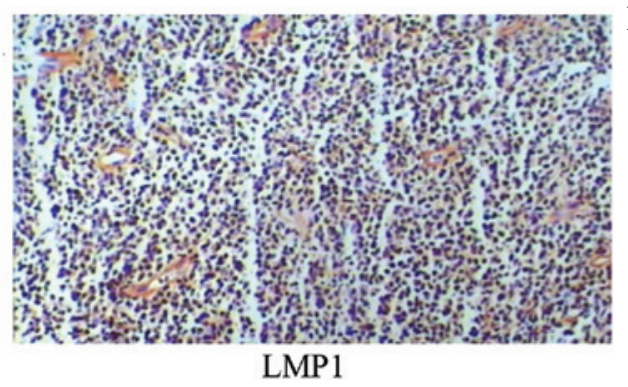

C

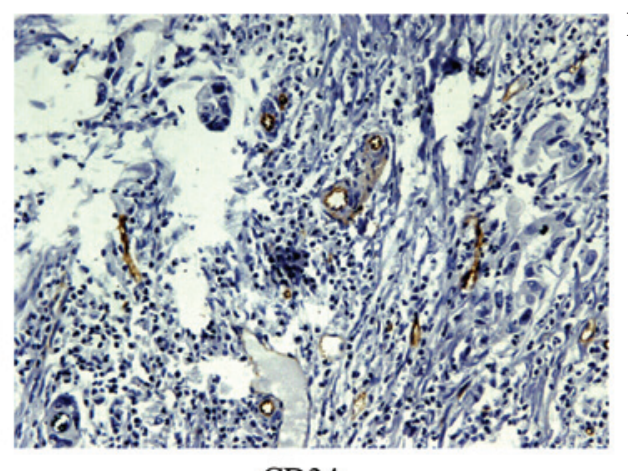

B
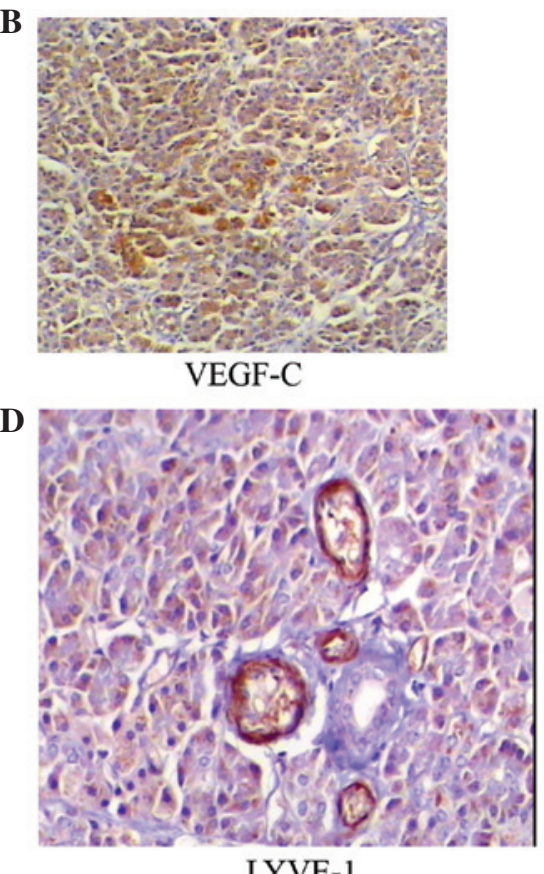

Figure 3. Expression of LMP1, VEGF-C, CD34 and LYVE-1 in nasopharyngeal tissues (x100 magnification). (A) Positive expression of LMP1; (B) positive expression of VEGF-C; (C) positive expression of CD34; and (D) positive expression of LYVE-1. LMP1, latent membrane protein 1; VEGF-C, vascular endothelial growth factor-C, LYVE-1, lymphatic vessel endothelial receptor 1.

demonstrated that EBV infection contributes to the development of a small percentage (5\%) of gastric cancers, although previous studies have shown that $10 \%$ of worldwide gastric cancers were EBVaGC $(6,20)$. By contrast, the frequency of EBV infection in NPC was notably higher, effecting $85 \%$ of the patients observed. Although the frequency of EBV infection in EBVaGC and NPC was different, the molecular mechanism of EBV infection in different types of cancer may be the same. Thus, in this study expression of the EBV-associated proteins LMP1 and BARF1 was further assessed in NPC and EBVaGC tissue specimens for association with clinicopathological data and with MVD and MLVD. It was demonstrated that BARF1 expression was associated with lymph node metastasis and MLVD in patients with EBVaGC. In NPC, LMP1 expression was associated with TNM stage $(\mathrm{P}=0.011)$ and lymph node metastasis $(\mathrm{P}=0.041)$. Only $13.3 \%$ cases were BARF1 positive and MLVD was significantly higher in
LMP1-positive cases than in LMP1-negative cases. The data from the current study indicate that although EBV infection is involved in the development of gastric cancer and NPC, expression of EBV-associated proteins LMP1 and BARF1 may have differential functions during the tumorigenesis of these two types of cancer.

Generally, primary EBV infection occurs via the oral route and establishes a lifelong virus carrier state, termed latent infection (21). In latent infection, infected cells only express a limited set of viral genes, but can provide a survival advantage to the infected cell (22). The latent infection can be further divided into different subgroups due to specific viral proteins (23). EBVaGC is considered as a latency I EBV infection, while NPC can be both latency I and II EBV infections $(24,25)$. Latency I infection is characterized by expression of EBV nuclear antigen 1 (EBNA1), EBER1 and 2, and BamHI-A rightward transcripts (BART) $(4,26)$. 
In addition to latency I transcripts, latency II infection can also express latent membrane protein 1 (LMP1) $(4,26)$. Our current data are consistent with previous findings $(4,26)$. Only one patient with weak LMP1 expression was identified in the 30 EBVaGC cases. By contrast, 38 NPC tissues in these 61 NPCs exhibited LMP1 expression. This confirmed that the latency of EBV infection between EBVaGC and NPC was different. Therefore, due to the different expression of viral proteins, oncogenic mechanisms of EBV in EBVaGC and NPC may differ.

Furthermore, tumor metastasis is the leading cause of cancer-related mortality $(27,28)$. A greater understanding of the molecular mechanism underlying tumor metastasis may aid the development of effective cancer therapies. The initial site of cancer metastasis is usually the regional lymph nodes (29,30). Clinical and experimental data suggested that lymphoangiogenesis can greatly facilitate the migration of tumor cells into the lymph nodes (31-33). The present study demonstrated that MLVD was associated with NPC lymph node metastasis and that EBV infection was associated with MLVD. The association between EBV infection and MLVD was consistent in EBVaGC and NPC. These data suggest that EBV infection may have a common effect on the regulation of lymphoangiogenesis, although the exact molecular mechanisms remain unknown. Further investigation is required to clarify how EBV infection contributes to an increase in MLVD.

EBV-positive epithelial malignancies show selective and abundant expression of a viral gene that encodes BARF1 protein (34). BARF1 expression was usually low in lymphomas, but more frequent in EBV-associated carcinomas. BARF1 may function as an oncogene in NPC, parallel to the more widely investigated viral protein LMP1 (35). In EBV-positive gastric cancer, BARF1 was expressed in the absence of LMP1, possibly functioning as the predominant EBV oncogene in this disease (36). BARF1 expression was able to immortalize and transform epithelial cells of different origins by acting as a mitogenic growth factor, inducing cyclin-D expression and upregulating anti-apoptotic Bcl-2, and in turn stimulating host cell growth and survival (37). In the current study, $13 \%$ of NPCs were positive for BARF1, whereas BARF1 was expressed in $56.7 \%$ of EBVaGCs. This finding suggests that BARF1 and LMP1 may have redundant functions in promoting tumorigenesis in gastric cancer and in NPC.

The current study does have certain limitations. For example, it is only a proof-of-principle descriptive study and additional mechanistic data are required to support the current findings. It remains to be determined how these two viral proteins function differentially in these two types of human cancer or whether EBV is involved in the development of EBV-positive gastric cancer since EBV is estimated to infect $>90 \%$ of the worldwide population and EBV infection may be just bystander in these gastric types of cancer.

\section{Acknowledgements}

This study was partially supported by the 973 Projects of China (grant no. 2011CB504302) and the Natural Science Foundation of China (grant no. 30872318). The authors would also like to acknowledge the valuable comments from other members of the laboratories.

\section{References}

1. Morales-Sánchez A and Fuentes-Pananá EM: Human viruses and cancer. Viruses 6: 4047-4079, 2014.

2. Xu X, Yang Z, Chen Q, Yu L, Liang S, Lü H and Qiu Z: Comparison of clinical characteristics of chronic cough due to non-acid and acid gastroesophageal reflux. Clin Respir J 9: 196-202, 2015.

3. Ok CY, Papathomas TG, Medeiros LJ and Young KH: EBV-positive diffuse large B-cell lymphoma of the elderly. Blood 122: 328-340, 2013.

4. Young LS and Rickinson AB: Epstein-Barr virus: 40 years on. Nat Rev Cancer 4: 757-768, 2004.

5. Tokunaga M, Land CE, Uemura Y, Tokudome T, Tanaka S and Sato E: Epstein-Barr virus in gastric carcinoma. Am J Pathol 143: 1250-1254, 1993.

6. Takada K: Epstein-Barr virus and gastric carcinoma. Mol Pathol 53: 255-261, 2000.

7. Pfitzner AJ, Tsai EC, Strominger JL and Speck SH: Isolation and characterization of $\mathrm{CDNA}$ clones corresponding to transcripts from the Bam $\mathrm{HI} \mathrm{H}$ and $\mathrm{F}$ regions of the Epstein-Barr virus genome. J Virol 61: 2902-2909, 1987.

8. Kelly GL, Long HM, Stylianou J, Thomas WA, Leese A, Bell AI, Bornkamm GW, Mautner J, Rickinson AB and Rowe M: An Epstein-Barr virus anti-apoptotic protein constitutively expressed in transformed cells and implicated in burkitt lymphomagenesis: The Wp/BHRF1 link. PLoS Pathog 5: e1000341, 2009.

9. Taub R, Kirsch I, Morton C, Lenoir G, Swan D, Tronick S, Aaronson S and Leder P: Translocation of the c-myc gene into the immunoglobulin heavy chain locus in human Burkitt lymphoma and murine plasmacytoma cells. Proc Natl Acad Sci USA 79: 7837-7841, 1982.

10. Adams JM, Harris AW, Pinkert CA, Corcoran LM, Alexander WS, Cory S, Palmiter RD and Brinster RL: The c-myc oncogene driven by immunoglobulin enhancers induces lymphoid malignancy in transgenic mice. Nature 318: 533-538, 1985.

11. Magrath I: The pathogenesis of Burkitt's lymphoma. Adv Cancer Res 55: 133-270, 1990.

12. Strasser A, Harris AW, Bath ML and Cory S: Novel primitive lymphoid tumours induced in transgenic mice by cooperation between myc and bcl-2. Nature 348: 331-333, 1990.

13. Beverly LJ and Varmus HE: MYC-induced myeloid leukemogenesis is accelerated by all six members of the antiapoptotic BCL family. Oncogene 28: 1274-1279, 2009.

14. Lee KM, Danuser R, Stein JV, Graham D, Nibbs RJ and Graham GJ: The chemokine receptors ACKR2 and CCR2 reciprocally regulate lymphatic vessel density. EMBO J 33: 2564-2580, 2014.

15. Hlatky L, Hahnfeldt P and Folkman J: Clinical application of antiangiogenic therapy: Microvessel density, what it does and doesn't tell us. J Natl Cancer Inst 94: 883-893, 2002.

16. Yates JL, Warren N and Sugden B: Stable replication of plasmids derived from Epstein-Barr virus in various mammalian cells. Nature 313: 812-815, 1985.

17. Gires O, Zimber-Strobl U, Gonnella R, Ueffing M, Marschall G, Zeidler R, Pich D and Hammerschmidt W: Latent membrane protein 1 of Epstein-Barr virus mimics a constitutively active receptor molecule. EMBO J 16: 6131-6140, 1997.

18. Dawson CW, Port RJ and Young LS: The role of the EBV-encoded latent membrane proteins LMP1 and LMP2 in the pathogenesis of nasopharyngeal carcinoma (NPC). Semin Cancer Biol 22: 144-153, 2012

19. Burke AP, Yen TS, Shekitka KM and Sobin LH: Lymphoepithelial carcinoma of the stomach with Epstein-Barr virus demonstrated by polymerase chain reaction. Mod Pathol 3: 377-380, 1990.

20. Shibata D and Weiss LM: Epstein-Barr virus-associated gastric adenocarcinoma. Am J Pathol 140: 769-774, 1992.

21. Shannon-Lowe $C$ and Rowe M: Epstein Barr virus entry; kissing and conjugation. Curr Opin Virol 4: 78-84, 2014.

22. Tsao SW, Tsang CM, Pang PS, Zhang G, Chen H and Lo KW: The biology of EBV infection in human epithelial cells. Semin Cancer Biol 22: 137-143, 2012.

23. Woellmer A and Hammerschmidt W: Epstein-Barr virus and host cell methylation: Regulation of latency, replication and virus reactivation. Curr Opin Virol 3: 260-265, 2013. 
24. Chen JN, He D, Tang F and Shao CK: Epstein-Barr virus-associated gastric carcinoma: A newly defined entity. J Clin Gastroenterol 46: 262-271, 2012.

25. Frappier L: Role of EBNA1 in NPC tumourigenesis. Semin Cancer Biol 22: 154-161, 2012.

26. Robertson KD, Manns A, Swinnen LJ, Zong JC, Gulley ML and Ambinder RF: CpG methylation of the major Epstein-Barr virus latency promoter in Burkitt's lymphoma and Hodgkin's disease. Blood 88: 3129-3136, 1996.

27. Lauria R, Perrone F, Carlomagno C, De Laurentiis M, Morabito A Gallo C, Varriale E, Pettinato G, Panico L, Petrella G, et al: The prognostic value of lymphatic and blood vessel invasion in operable breast cancer. Cancer 76: 1772-1778, 1995.

28. Kyzas PA, Geleff S, Batistatou A, Agnantis NJ and Stefanou D: Evidence for lymphangiogenesis and its prognostic implications in head and neck squamous cell carcinoma. J Pathol 206: 170-177, 2005.

29. Chua B, Ung O, Taylor R and Boyages J: Frequency and predictors of axillary lymph node metastases in invasive breast cancer. ANZ J Surg 71: 723-728, 2001.

30. Cunnick GH, Jiang WG, Gomez KF and Mansel RE: Lymphangiogenesis and breast cancer metastasis. Histol Histopathol 17: 863-870, 2002.

31. Skobe M, Hawighorst T, Jackson DG, Prevo R, Janes L, Velasco P, Riccardi L, Alitalo K, Claffey K and Detmar M: Induction of tumor lymphangiogenesis by VEGF-C promotes breast cancer metastasis. Nat Med 7: 192-198, 2001.
32. He Y, Karpanen T and Alitalo K: Role of lymphangiogenic factors in tumor metastasis. Biochim Biophys Acta 1654: 3-12, 2004

33. Salven P, Mustjoki S, Alitalo R, Alitalo K and Rafii S: VEGFR-3 and CD133 identify a population of CD34+ lymphatic/vascular endothelial precursor cells. Blood 101: 168-172, 2003.

34. Brink AA, Vervoort MB, Middeldorp JM, Meijer CJ and van den Brule AJ: Nucleic acid sequence-based amplification, a new method for analysis of spliced and unspliced Epstein-Barr virus latent transcripts, and its comparison with reverse transcriptase PCR. J Clin Microbiol 36: 3164-3169, 1998.

35. Zheng H, Li LL, Hu DS, Deng XY and Cao Y: Role of Epstein-Barr virus encoded latent membrane protein 1 in the carcinogenesis of nasopharyngeal carcinoma. Cell Mol Immunol 4: 185-196, 2007.

36. zur Hausen A, Brink AA, Craanen ME, Middeldorp JM, Meijer CJ and van den Brule AJ: Unique transcription pattern of Epstein-Barr virus (EBV) in EBV-carrying gastric adenocarcinomas: Expression of the transforming BARF1 gene. Cancer Res 60: 2745-2748, 2000.

37. Chang MS, Kim DH, Roh JK, Middeldorp JM, Kim YS, Kim S, Han S, Kim CW, Lee BL, Kim WH and Woo JH: Epstein-Barr virus-encoded BARF1 promotes proliferation of gastric carcinoma cells through regulation of NF-kB. J Virol 87: 10515-10523, 2013. 